\title{
Dynamics and 'normal stress' evaluation of dilute suspensions of periodically forced prolate spheroids in a quiescent Newtonian fluid at low Reynolds numbers
}

\author{
K MADHUKAR $^{1,3}$, P V KUMAR $^{2}$, T R RAMAMOHAN ${ }^{1}$ \\ and I S SHIVAKUMARA ${ }^{3}$ \\ ${ }^{1}$ Centre for Mathematical Modelling and Computer Simulation (C-MMACS), \\ Council of Scientific and Industrial Research (CSIR), Wind Tunnel Road, \\ Bangalore 560037 \\ ${ }^{2}$ Department of Metallurgical and Materials Engineering, Indian Institute of \\ Technology Madras, Chennai 600036 \\ ${ }^{3}$ Department of Mathematics, UGC-Centre for Advanced Studies in Fluid \\ Mechanics, Bangalore University, Bangalore 560001 \\ e-mail:k.madhukara@gmail.com, priyank022@gmail.com, \\ trr@cmmacs.ernet.in, shivakumarais@gmail.com
}

MS received 24 September 2009; revised 3 July 2010; accepted 6 August 2010

\begin{abstract}
The problem of determining the force acting on a particle in a fluid where the motion of the fluid and the particle is given has been considered in some detail in the literature. In this work, we propose an example of a new class of problems where, the fluid is quiescent and the effect of an external periodic force on the motion of the particle is determined at low non-zero Reynolds numbers. We present an analysis of the dynamics of dilute suspensions of periodically forced prolate spheroids in a quiescent Newtonian fluid at low Reynolds numbers including the effects of both convective and unsteady inertia. The inclusion of both forms of inertia leads to a nonlinear integro - differential equation which is solved numerically for the velocity and displacement of the individual particle. We show that a 'normal stress' like parameter can be evaluated using standard techniques of Batchelor. Hence this system allows for an experimentally accessible measurable macroscopic parameter, analogous to the 'normal stress', which can be related to the dynamics of individual particles. We note that this 'normal stress' arises from the internal fluctuations induced by the periodic force. In addition, a preliminary analysis leading to a possible application of separating particles by shape is presented. We feel that our results show possibilities of being technologically important since the 'normal stress' depends strongly on the controllable parameters and our results may lead to insights in the development of active dampeners and smart fluids. Since we see complex behaviour even in this simple system, it is expected that the macroscopic behaviour of such suspensions may be much more complex in more complex flows.
\end{abstract}


Keywords. Low Reynolds number; quiescent fluid; periodically forced prolate spheroids; aspect ratio; inertial effects and particle separation.

\section{Introduction}

Suspensions of solid particles are encountered both as raw materials and as intermediates in a large number of industries such as printing and paper making, petroleum processing, pharmaceuticals and food processing. In most situations, the particles tend to be non-spherical or even irregularly shaped, the suspension rheology then being sensitive to the orientation distribution of the suspended particles. The motion of non-spherical particles in shear flows at vanishingly small Reynolds numbers has been studied theoretically for a long time and the literature in this case is summarized by Leal (1980). It has, in fact been known since the work of Jeffery (1922) and later Bretherton (1962), that in the absence of inertia, an axisymmetric particle in a simple shear flow rotates periodically in one of an infinite single-parameter family of closed 'Jeffery' orbits. The particular orbit adopted by the particle, in the absence of hydrodynamic interactions, Brownian motion, etc. depends on the initial conditions, rendering the inertialess limit indeterminate. Subramanian \& Koch (2006) considered both particle and fluid inertia as a possible mechanism acting to remove this indeterminancy. They developed solutions for aspect ratios close to unity. Hence, their analysis captures the leading order effect of the deviation from sphericity on the particle orientational motion. They found that for the neutrally buoyant case, the inertia of the suspending fluid causes a prolate spheroid to drift toward an axial spin about the vorticity axis of the ambient simple shear. This suggests that inertial effects play a major role in the dynamics of a particle and hence forms one motivation for our work.

It is also interesting to determine the dynamics and rheology of dipolar particles in the presence of an external electric or magnetic field, which could lead to possible applications in electrorheological and magnetorheological fluids. Literature in this regard has been compiled by Strand \& Kim (1992). They have investigated the rheological and rheo-optical properties of dilute suspensions of Brownian particles having permanent dipoles subject to time-dependent shear and external fields. A wide range of demonstrated applications such as separation processes, catalytic reactors, etc. have been compiled by Rosensweig (1985). Other practical applications of the study of the dynamics of small dipolar particles in various linear flows under the effect of alternating or rotating external fields include magnetofluidization (Buevich et al 1984), magnetostriction of ferromagnetic particle suspensions (Ignatenko et al 1984), characterization of magneto rheological suspensions (Cebers 1993) and determining the rheological properties of ferromagnetic colloids (Tsebers 1986).

In addition, a number of authors have considered the evaluation of the force acting on a body whose motion is known at low non-zero Reynolds numbers (Lovalenti \& Brady 1993a, 1995). Since the motion of the particle and fluid are known, the evaluation of the history term is relatively elementary. There is also an indication that history forces are not negligible even at large Reynolds numbers (Gondret et al 2002).

In this paper, we present the inverse problem, namely the effect of a periodic force on the motion of a dilute suspension of prolate spheroidal particles in a quiescent Newtonian fluid at low non-zero Reynolds numbers. We hope to isolate the effects of particle shape on the dynamics of the particle at low non-zero Reynolds number in the simplest possible case. We also calculate a 'Normal stress' like macroscopic experimentally accessible parameter using standard techniques following Batchelor (1970). This experimentally accessible parameter 
can be related to the dynamics of individual particles and hence this system could be considered as a system wherein macroscopic behaviour can be related to microscopic behaviour. This class of problems is one of the simplest physically realizable fluid dynamical systems that can show nonlinear behaviour at the level of the individual particle. There is a nonlinear coupling between the scale of the individual particle and the scale of the macroscopic parameter through the time dependent force. This system is thus an ideal system to probe the statistical mechanics of systems consisting of a large number of periodically driven oscillators with a fading memory. It has been shown that there exists a chaotic parametric regime, in the dynamics of periodically forced spheroidal particles in a simple shear flow (Kumar et al 1995). This chaotic dynamics can be controlled by controlling system parameters (Kumar \& Ramamohan 1998). These results restricted to zero Reynolds numbers and simple shear flows have been summarized by Asokan et al (2005).

Recently, Ramamohan et al (2009) have studied the dynamics of a dilute suspension of neutrally buoyant periodically forced spherical particles in a quiescent Newtonian fluid at low Reynolds numbers. This represents the first step to extend the results summarized by Asokan et al (2005), to the low Reynolds number regime. In this paper, we extend these results to prolate spheroidal particle suspensions as a prelude to a study of more complex suspensions.

\section{The hydrodynamic force expression for an arbitrary shaped particle}

Lovalenti \& Brady (1993) have given the expression for the required hydrodynamic force on an arbitrary shaped particle, in the long time limit at low Reynolds numbers. The reciprocal theorem has been used to obtain the following expression. The details of the derivation can be found in Lovalenti \& Brady (1993).

$$
\begin{aligned}
F^{H}(t)= & \operatorname{ReSl} V_{p} \dot{\boldsymbol{U}}^{\infty}(t)+\boldsymbol{F}_{s}^{H}(t) \\
& -\operatorname{ReSl}\left[6 \pi \boldsymbol{\phi} \cdot \boldsymbol{\phi} \cdot \boldsymbol{\phi}+\lim _{R \rightarrow \infty}\left(\int_{V_{F}(R)} \boldsymbol{M}^{T} \cdot \boldsymbol{M} d V-\frac{9 \pi}{2} \boldsymbol{\phi} \cdot \boldsymbol{\phi} R\right)\right] \cdot \dot{\boldsymbol{U}}_{s}(t) \\
& +\frac{3}{8}\left(\frac{\operatorname{ReSl}}{\pi}\right)^{1 / 2}\left\{\int _ { - \infty } ^ { t } \left[\frac{2}{3} \boldsymbol{F}_{s}^{H_{\|}}(t)-\left\{\frac{1}{|\boldsymbol{A}|^{2}}\left(\frac{\pi^{1 / 2}}{2|\boldsymbol{A}|} \operatorname{erf}(|\boldsymbol{A}|)-\exp \left(-|A|^{2}\right)\right)\right\} \boldsymbol{F}_{s}^{H_{\|}}(s)\right.\right. \\
& \left.+\frac{2}{3} \boldsymbol{F}_{s}^{H_{\perp}}(t)-\left\{\exp \left(-|\boldsymbol{A}|^{2}\right)-\frac{1}{2|\boldsymbol{A}|^{2}}\left(\frac{\pi^{1 / 2}}{2|\boldsymbol{A}|} \operatorname{erf}(|\boldsymbol{A}|)-\exp \left(-|\boldsymbol{A}|^{2}\right)\right)\right\} \boldsymbol{F}_{s}^{H_{\perp}}(s)\right] \\
& \left.\times \frac{2 d s}{(t-s)^{3 / 2}}\right\} \cdot \phi \\
& -\operatorname{Relim}_{R \rightarrow \infty} \int_{V_{F}(R)}\left(\boldsymbol{u}_{0} \cdot \nabla \boldsymbol{u}_{0}-\boldsymbol{U}_{s}(\boldsymbol{t}) \cdot \nabla \boldsymbol{u}_{0}\right) \cdot \boldsymbol{M}+o(\operatorname{ReSl})+o(\operatorname{Re}) .
\end{aligned}
$$

Here, $\boldsymbol{U}_{s}=\boldsymbol{U}_{p}-\boldsymbol{U}^{\infty}$ is the slip velocity of the fluid. $\boldsymbol{U}_{p}$ is the velocity of the particle. $\boldsymbol{U}_{s}$ has been non-dimensionalized by $U_{c}$. The acceleration terms $\dot{\boldsymbol{U}}_{s}$ and $\dot{\boldsymbol{U}}^{\infty}$ are non-dimensionalized by $\omega U_{c}$, where $1 / \omega$ is the characteristic timescale. $\boldsymbol{U}^{\infty}$ is the velocity of the fluid as $r \rightarrow \infty$. $\operatorname{Re}$ is the Reynolds number, defined as $\operatorname{Re}=U_{c} a / v$ based on a characteristic particle slip velocity, $U_{c}$, ' $a$ ' denotes the characteristic particle dimension, in our case the semi-major axis and $v$ is the kinematic viscosity of the fluid. $F_{s}^{H}=-6 \pi\left(\phi \cdot U_{s}\right), F_{s}^{H_{\|}}=-6 \pi\left(\phi \cdot U_{s}\right) \cdot(p p)$, $F_{s}^{H_{\perp}}=-6 \pi\left(\phi \cdot U_{s}\right) \cdot(\delta-p p)$, where $\delta$ is the idem tensor of order 2 and unit vector 
Table 1. Computed values of the diagonal matrix representing the acceleration reaction term.

\begin{tabular}{llll}
\hline Aspect ratio & $I_{x x}$ & $I_{y y}$ & $I_{z z}$ \\
\hline 2 & $4 \cdot 62$ & $9 \cdot 2$ & $9 \cdot 2$ \\
3 & $6 \cdot 45$ & $17 \cdot 0$ & $17 \cdot 0$ \\
4 & $8 \cdot 50$ & $25 \cdot 0$ & $25 \cdot 0$ \\
5 & $10 \cdot 34$ & $33 \cdot 05$ & $33 \cdot 05$ \\
6 & $11 \cdot 73$ & $38 \cdot 05$ & $38 \cdot 05$ \\
7 & $12 \cdot 30$ & $40 \cdot 5$ & $40 \cdot 5$ \\
8 & $14 \cdot 85$ & $47 \cdot 0$ & $47 \cdot 0$ \\
9 & $19 \cdot 25$ & $59 \cdot 5$ & $59 \cdot 5$ \\
10 & $19 \cdot 30$ & 61.5 & $61 \cdot 5$ \\
\hline
\end{tabular}

$\boldsymbol{p}=\frac{\boldsymbol{Y}_{\boldsymbol{s}}(\boldsymbol{t})-\boldsymbol{Y}_{\boldsymbol{s}}(\boldsymbol{s})}{\left|\boldsymbol{Y}_{\boldsymbol{s}}(\boldsymbol{t})-\boldsymbol{Y}_{\boldsymbol{s}}(\boldsymbol{s})\right|}$, here $\boldsymbol{Y}_{s}(t)-\boldsymbol{Y}_{s}(s)$ is the integrated displacement of the particle relative to the fluid from time $s$ to the current time $t . F^{H}$ is scaled by $\mu a U_{c}$. Sl is the Strouhal number and ' $\boldsymbol{A}$ ' is given by

$$
A=\frac{\operatorname{Re}}{2}\left(\frac{t-s}{\operatorname{ReS} 1}\right)^{1 / 2}\left(\frac{Y_{s}(t)-Y_{s}(s)}{t-s}\right) .
$$

The first term on the right hand side of the differential expression is due to an accelerating reference frame. The second is the pseudo-steady Stokes drag. The third is called the acceleration reaction, similar to the added mass. The fourth term represents the unsteady Oseen correction, which replaces the 'Basset memory integral' in the long time limit at finite Reynolds number. The last integral contributes a lift force, i.e. a force perpendicular to the slip velocity. We note that the expression is valid up to order Re and order ReSl.

The acceleration reaction term ( $I_{x x}$ in table 1$)$ is computed using the expressions given by Pozrikidis (1992) and Chwang \& Wu (1975). The expression for the Stokes resistance tensor $(\phi)$ in its dimensionless form is given by

$$
\phi=\frac{8 e}{3}(a)
$$

Here, $e$ is the eccentricity of the spheroid and $\boldsymbol{a}$ is a tensor depending on the geometry of the particle given by

$$
a=\left[\begin{array}{ccc}
e_{1} & 0 & 0 \\
0 & e_{2} & 0 \\
0 & 0 & e_{2}
\end{array}\right]
$$

where,

$$
\begin{aligned}
& e_{1}=\frac{e^{2}}{\left[-2 e+\left(1+e^{2}\right) \log \left(\frac{1+e}{1-e}\right)\right]} \\
& e_{2}=\frac{-2 e^{2}}{\left[-2 e+\left(1-3 e^{2}\right) \log \left(\frac{1+e}{1-e}\right)\right]}
\end{aligned}
$$




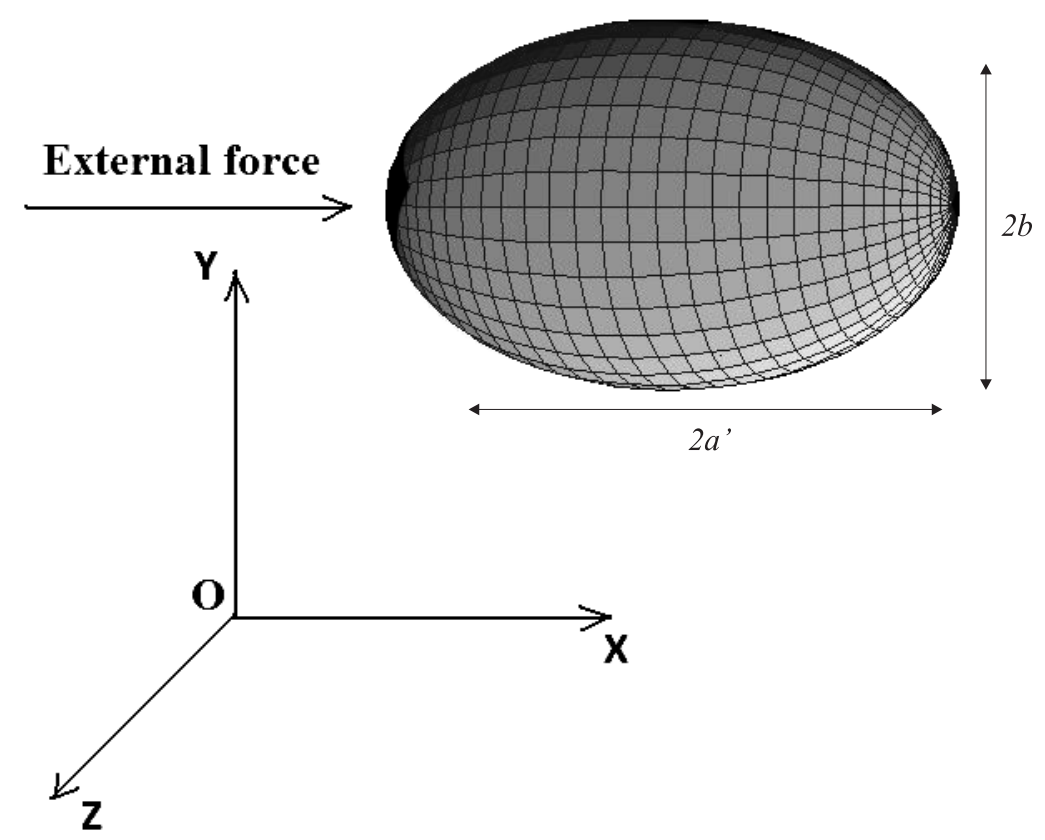

Figure 1. Schematic representation of an external force acting on a prolate spheroidal particle along the $x$-axis.

In the current work as a preliminary, we deal only with a one-dimensional motion of the particle. The translation is along the major axis of the spheroid and hence is symmetric with respect to the particle. Therefore, we must neglect the lift force term as it contributes only to a force in the perpendicular direction and is zero in this case. This has been verified in our computations. We note that the lift force typically manifests itself in the motion of particles near a wall or when there is more than one direction in the problem.

\section{Solving the differential equation}

After obtaining suitable values of the remaining integrals in (1) for different aspect ratios, we determined the dynamics of the spheroidal particle. From the motion of the particle, we determined the macroscopic parameters using standard expressions.

\subsection{Formulation of the problem}

We consider the force equation (1) given by Lovalenti and Brady for an arbitrary shaped particle undergoing an arbitrary time-dependent motion at low Reynolds numbers, in the long time limit. In our case we consider a neutrally buoyant prolate spheroid in an infinite body of quiescent fluid and consider the effects of an external periodic force acting on the spheroid along the $x$-axis as shown in figure 1 .

We use equation (1) to obtain the governing expression for the unidirectional motion of a spheroid in a quiescent fluid medium, starting with zero velocity and displacement at time $t=0$, with $\boldsymbol{U}_{s}=\boldsymbol{U}_{p}-\boldsymbol{U}^{\infty}$ where $\boldsymbol{U}_{p}$ is the velocity of the particle, scaled with respect to the size of the particle $a^{\prime}$ (see figure 1) and the frequency of the external periodic force, $\omega$, i.e. we take $U_{c}=a^{\prime} \omega$ and $\boldsymbol{U}^{\infty}=0$. 
We note that there exists a singularity at the point $s=t$. In order to avoid this singularity, we evaluated the integral in the interval $[0, t-\epsilon]$, where $\epsilon$ is chosen to be a very small number. We note that in the limit $s \rightarrow t$, the integral converges to a finite limit and hence the value of the integral in the range $s=t-\epsilon$ to $s=t$ is negligible. Under these conditions, equation (1) reduces to

$$
\begin{aligned}
\boldsymbol{F}^{\boldsymbol{H}}(t)= & -6 \pi(\operatorname{cof}) U_{p}(t)-\operatorname{ReSl}\left(I_{x x}\right) \dot{\boldsymbol{U}}_{\boldsymbol{s}}(\boldsymbol{t})+\frac{3}{8}\left(\frac{\operatorname{ReS} l}{\pi}\right)^{1 / 2}(\operatorname{cof})^{2} \\
& \times\left\{\int_{0}^{t-\varepsilon}\left\{\frac{1}{|\boldsymbol{A}|^{2}}\left(\frac{\pi^{1 / 2}}{2|\boldsymbol{A}|} \operatorname{erf}(|\boldsymbol{A}|)-\exp \left(-|\boldsymbol{A}|^{2}\right)\right)\right\} \frac{12 \pi \boldsymbol{U}_{p}(s)}{(t-s)^{3 / 2}} d s\right. \\
& \left.+16 \pi U_{p}(t)\left[\frac{1}{\sqrt{t}}-\frac{1}{\sqrt{\varepsilon}}\right]\right\} .
\end{aligned}
$$

Here, $c o f=\frac{8}{3} e a_{11}$ and $I_{x x}$ is the computed acceleration reaction term (see table 1).

The equation of motion for a neutrally buoyant particle immersed in a liquid is given by

$$
\frac{m_{p} \dot{\boldsymbol{U}}_{p}(\boldsymbol{t})}{\mu a^{2} \omega}=\boldsymbol{F}^{\mathrm{ext}}(\boldsymbol{t})+\boldsymbol{F}^{\boldsymbol{H}}(\boldsymbol{t}) .
$$

We use the periodic force $F^{\text {ext }}(t)=F_{0} \sin (t)$, where time has been scaled with respect to the frequency of the external periodic force. We get the following equations for the $x$ component of the displacement and velocity of the particle using the Newton's second law of motion.

$$
\begin{aligned}
\frac{d Y_{p}}{d t} & =U_{p} . \\
\frac{d U_{p}}{d t} & =\frac{1}{\operatorname{Re}^{\prime}}\left[\operatorname{Re}_{F} \sin (t)-6 \pi(\operatorname{cof}) U_{p}+\frac{3}{8}\left(\frac{\operatorname{ReSl}}{\pi}\right)^{1 / 2}(\operatorname{cof})^{2}\left(P_{1}+Q_{1}\right)\right] \\
\operatorname{Re}^{\prime} & =\frac{4 \pi}{3}\left(\frac{b}{a^{\prime}}\right)^{2} \operatorname{Re}+I_{x x} \operatorname{ReSl}, \quad \operatorname{Re}_{F}=\frac{F_{0}}{\mu a^{2} \omega}, \operatorname{Re}=\frac{\rho a^{2} \omega}{\mu}
\end{aligned}
$$

Here, $a^{\prime}$ is the characteristic particle dimension, $\rho$ is the density of the particle and $\mu$ is the fluid viscosity.

$$
\begin{aligned}
P_{1} & =\int_{0}^{t-\varepsilon}\left\{\frac{1}{|A|^{2}}\left(\frac{\pi^{1 / 2}}{2|A|} \operatorname{erf}(|A|)-\exp \left(-|A|^{2}\right)\right)\right\} \frac{12 \pi U_{p}(s)}{(t-s)^{3 / 2}} d s \\
Q_{1} & =16 \pi U_{p}(t)\left[\frac{1}{\sqrt{t}}-\frac{1}{\sqrt{\varepsilon}}\right] .
\end{aligned}
$$

We developed software using Numerical Recipes in FORTRAN 77 (Press et al 1992) to solve the differential equations using an embedded Runge-Kutta method with adaptive step size. The integral in the equation (6) was evaluated at each time step by Romberg extrapolation. The function with respect to ' $\boldsymbol{A}$ ' ' was defined by a user supplied function subprogram. We used the ODEINT, RKQS, RKCK subroutines from Numerical Recipes to implement the RungeKutta method. The Romberg extrapolation was performed using the QROMB subroutine. The integral was evaluated using TRAPZD and the interpolation during the numerical quadrature 
was performed by POLINT. The tolerance for both the Romberg extrapolation and the RungeKutta solver was taken as $10^{-5}$. Further reduction of the tolerance did not result in any significant change in our results. The entire program was written in double precision. The initial conditions for both the velocity and the position of the particle were taken as zero. $\varepsilon$ was taken as 0.04 ; smaller values of $\varepsilon$ did not significantly change the results. The software was tested for consistency by compiling the program with two compilers namely, Intel Fortran and F90. We generated 5000 data points taken at an interval of $\pi / 400$ in both the dimensionless velocity and dimensionless position. Further increase in the resolution did not yield any difference in our results.

\subsection{Tests}

We performed several tests in order to validate the results obtained. They are listed below.

3.2a Test 1-perturbation analysis: We obtained perturbation solutions in order to validate our results for small Re. We used a Taylor series expansion for the nonlinear integral term and included only the first linear term. One important aspect to be noted is that the expression given for an arbitrary shaped particle (prolate spheroid in our case) is correct up to $\mathrm{O}(\mathrm{ReSl})$.

The perturbation parameter was chosen as $\mathrm{Re}^{1 / 2}$. The hydrodynamic force expression for an arbitrary shaped particle given by Lovalenti and Brady is valid up to $\mathrm{O}(\mathrm{Re})$.

Hence, we express our perturbed solution up to $\mathrm{O}(\mathrm{Re})$ as follows.

$$
U_{p}=U_{0}+U_{1} \operatorname{Re}^{1 / 2}+U_{2} \operatorname{Re}+o(\operatorname{Re}),
$$

where

$$
\begin{aligned}
& U_{0}=\frac{\operatorname{Re}_{F} \sin (t)}{6 \pi(\operatorname{cof})} \\
& U_{1}=\frac{Q U_{0}}{6 \pi(\operatorname{cof})}\left[\frac{1}{\sqrt{t}}-\frac{1}{\sqrt{\varepsilon}}\right]+\frac{3 \operatorname{Re}_{F}(\operatorname{Sl} \pi)^{1 / 2}}{(6 \pi)^{2}} \int_{0}^{t-\varepsilon} \frac{\sin (s)}{(t-s)^{3 / 2}} d s \\
& U_{2}=\frac{-1}{6 \pi(\operatorname{cof})}\left\{\frac{c_{1} \operatorname{Re}_{F} \cos (t)}{6 \pi(\operatorname{cof})}-Q U_{1}\left[\frac{1}{\sqrt{t}}-\frac{1}{\sqrt{\varepsilon}}\right]-3(\mathrm{~S} 1 \pi)^{1 / 2}(\operatorname{cof})^{2} \int_{0}^{t-\varepsilon} \frac{U_{1}(s)}{(t-s)^{3 / 2}} d s\right\},
\end{aligned}
$$

where in

$$
Q=6(\mathrm{Sl} \pi)^{1 / 2}(\operatorname{cof})^{2}, c_{1}=\frac{4 \pi}{3}\left(\frac{b}{a^{\prime}}\right)^{2}+\left(I_{x x}\right) \mathrm{S} 1 .
$$

The displacement is calculated by numerically integrating the interpolated data of the velocity.

MATLAB was used for computing the above expressions. The integrals were evaluated using the trapezoidal rule through the trapz function, while the cubic interpolation technique was employed for displacement calculations. We found that for low values of Re, typically up to $\operatorname{Re}=0 \cdot 05$, both the perturbed and the numerical solutions agreed well. Figure 2 shows a comparison between the plots obtained by both the methods for aspect ratios 2, 6 and 10, $\operatorname{Re}=0.03$ and $\operatorname{Re}_{F}=0.1$.

Here, we observe that as aspect ratio increases, the effect of inertia decreases and hence the perturbation solution is closer to the numerical solution. This is due to the fact that we include only the linearized form of the convective inertia term in the calculation of the perturbation solution. 

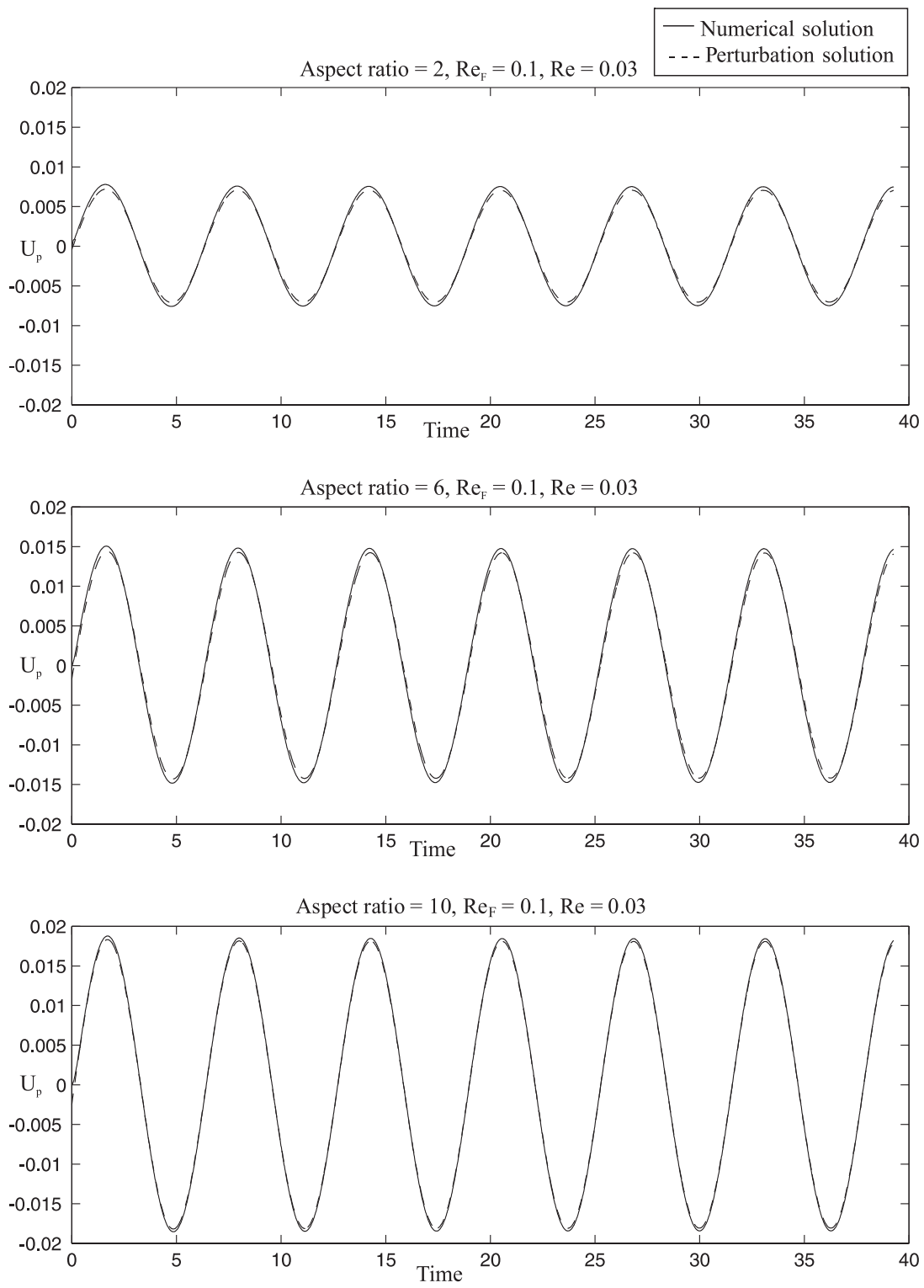

Figure 2. The comparison of the numerical solution with the perturbation solution for $\mathrm{Re}=0.03, \operatorname{Re}_{F}=0 \cdot 1$, aspect ratio $=2,6$ and 10 . We see that the match is good with the increase in aspect ratio owing to reduced inertial effects at higher aspect ratios and hence reduced nonlinearity.

3.2b Test 2: We reproduced the curve obtained by Lovalenti and Brady for the sedimentation problem of a spherical particle (the result is shown in figure 3 ).

3.2c Test 3: When the initial direction of the motion was reversed, namely by replacing $\operatorname{Re}_{F}$ with $-\operatorname{Re}_{F}$, the phase space plot was reflected about the zero velocity axis. That is, a reflection of the phase space attractor about the zero velocity axis when the direction of the first motion is reversed was obtained, which can be considered as an important result which 


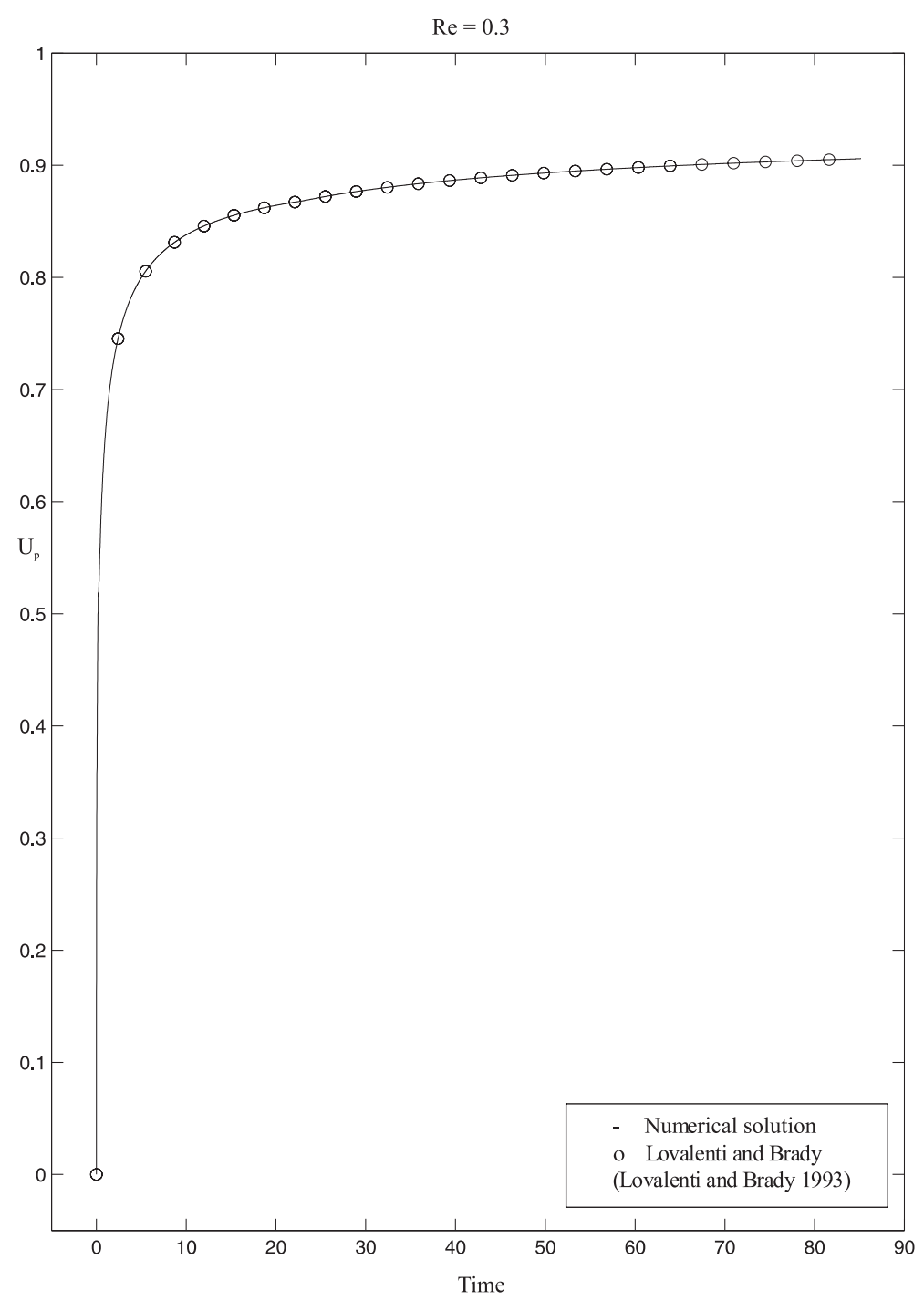

Figure 3. Comparison of the curves obtained for the sedimentation problem of a spherical particle by Lovalenti and Brady and the present work as a test for our numerical procedure adopted. In this case, $\operatorname{Re}=0 \cdot 3$.

demonstrates the correctness of the results. The results showed a preferred direction in the solution. Since the only physical direction in our present problem is the initial direction of the external force, a reversal of that direction should result in a reversal of direction in the solution, which was indeed the case.

3.2d Test 4: When the initial condition of $Y_{P}$ was changed, we observed a shift in the position of the attractor, i.e. there was merely a shift in the position about which the particle was found to oscillate. Changing $Y_{P}$ at $t=0$ shifts the attractors without affecting the physics of the problem and this was verified by our test. The tests performed gave considerable confidence in our results. 


\section{Results and discussions}

We have four variable parameters in the system; the Reynolds number Re, the Strouhal number $\mathrm{Sl}$, the aspect ratio and the amplitude of the periodic force $\mathrm{Re}_{F}$. It is essential to determine the effect of these parameters on the system as well as on the 'normal stress'. Typical phase space plots (plots of particle velocity vs. position) have been generated for different values of the Reynolds number, the aspect ratio and the amplitude of the periodic force. We choose to keep one of the parameters namely the Strouhal number, a constant and equal to unity. The plots represent an attractor as they are bounded in phase space. Since S1 always occurs in combination with $\mathrm{Re}$ in the equation, the limit of small Sl number is automatically obtained by reducing Re. In order to account for the drift in the preferred direction in the problem, we determined the average displacement of the particle from the zero-position axis, denoted as $Y_{\text {pmean }}$.

The area bounded by the phase space plot which is bounded and hence represents an attractor in phase space, increases with increasing amplitude of the forcing term, $\mathrm{Re}_{F}$, establishing the obvious relation between the attractors and the amplitude of the periodic force. As $\operatorname{Re}_{F}$ increases, the particle oscillates with larger amplitude and thus covers a larger surface area in the phase plot. As can be seen from figure 4, the increase in area is quite significant when $\operatorname{Re}_{F}$ is increased from 0.01 to 0.05 . This shows that the periodic force, which initiates the motion of the particle, has a strong influence on the particle's velocity and displacement. We have also shown the effect of $\operatorname{Re}_{F}$ on increasing the aspect ratio. The influence is much greater and the attractor covers relatively greater surface area. The reason being, at higher aspect ratios, the value of the components of the Stokes Resistance tensor are lower. This result suggests that the displacement of the particle increases with aspect ratio (shape) and hence this indicates a possibility that we can separate particles of different shapes using a periodic forcing in a quiescent fluid.

The effect of increasing the Reynolds number can be seen from figure 5. The effect is opposite compared to that of increasing the amplitude of the forcing term. Increasing Re results in a smaller attractor plot. The surface area covered by the particle decreases in the phase plot. This shows the effect of inertia on the motion of the particle. Inertial effects dominate at higher Reynolds number and the mean position of the particle is seen to shift in the direction of initial motion on increasing Re. On comparing the results obtained for different aspect ratios, we find that this shift is more significant when the aspect ratio is larger. In figure 5 there are kinks in the lower aspect ratio regime which vanish as aspect ratio increases. These kinks are present only for aspect ratios up to 2 and $\operatorname{Re}_{F}=0.01$. We tried to eliminate these kinks by changing the parameters of the numerical algorithm; however they persisted under all the changes we made in the tolerance and the resolution of the solution. Hence we feel that they are an integral part of our solution. Since solutions of nonlinear equations have been known to contain discontinuities, we feel that they are a part of the solution. We had observed similar kinks in the calculation of the phase plots for the sphere problem (Ramamohan et al 2009). At $\operatorname{Re}_{F}=0 \cdot 01$, inertial effects dominate. When the displacement of the particle is a maximum or a minimum, we observe that the velocity of the particle is near to zero and its acceleration is at a maximum and at this mean position at low $\operatorname{Re}_{F}$, the particle may behave a little jerkily due to the effect of inertia. In figure 4, it is shown that increasing Re leads to a decrease in the surface area of the phase space plots. Increase in Re implies that the resistance to change in motion is high and hence the distance traversed by the particle reduces due to this. In figures 4 and 5, we see that the particle tends to drift away from the zero position axis with each cycle. We see that at higher aspect ratios the phase plots are larger in size. 

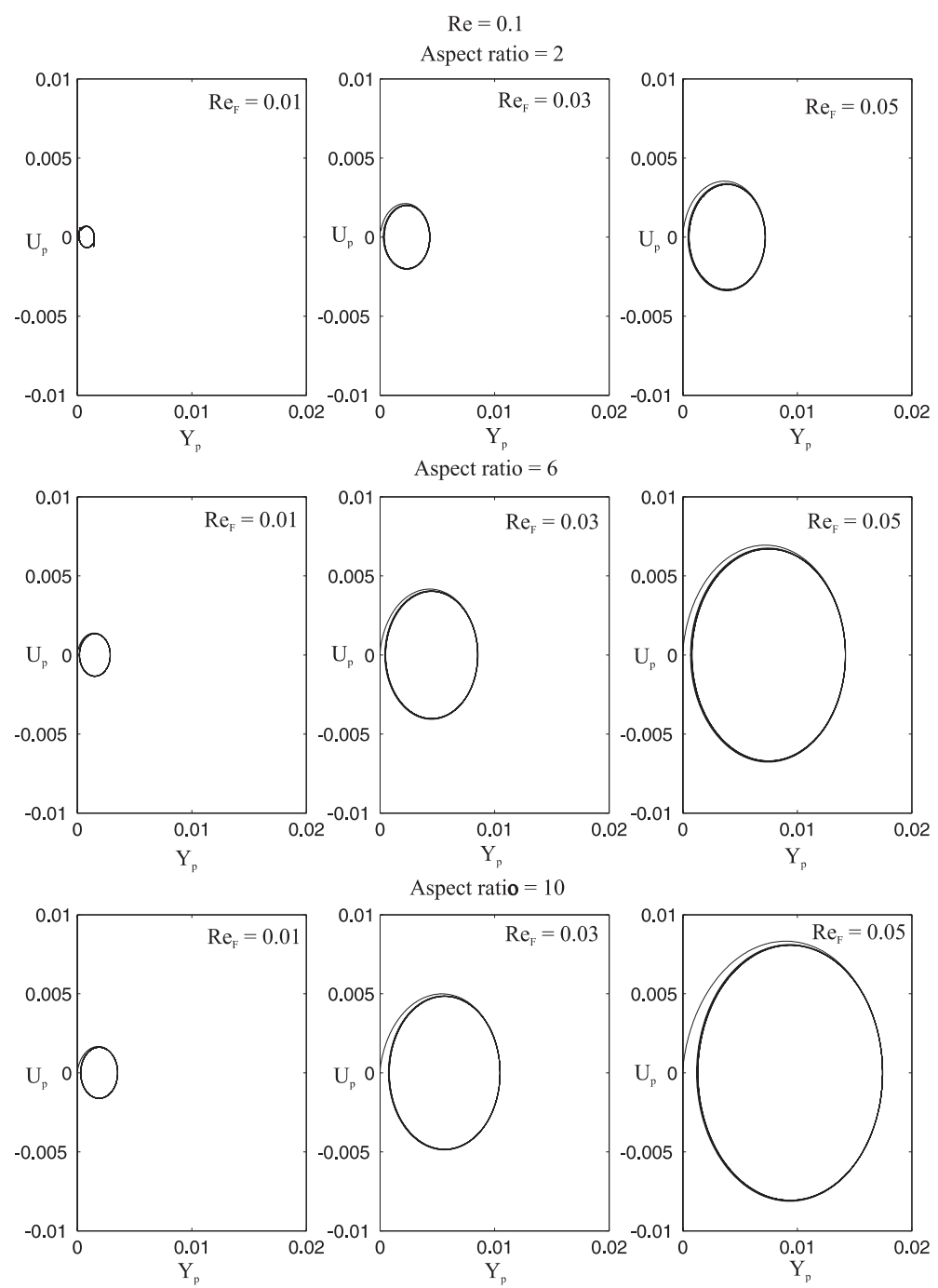

Figure 4. This phase portrait shows the effect of $\operatorname{Re}_{F}$ on the system. We see that on increasing the value of $\operatorname{Re}_{F}$, the phase plots get enlarged showing the obvious effect of the forcing term. The phase plots for different $\operatorname{Re}_{F}$ values $(0 \cdot 01,0 \cdot 03,0 \cdot 05), \operatorname{Re}=0 \cdot 1$, aspect ratio $=2,6$ and 10 .

It can be seen that in the initial cycles, the drift is significant when compared to the drift in the later stages of the simulation. Thus, we see that the inertial effects are dominant during the initial stages and later on the particle tends towards an oscillatory steady state. The effect of the aspect ratio can also be seen here. For particles with lower aspect ratios, inertial effects coupled with higher resistance cause the phase plot to be smaller in size, whereas for the particles with higher aspect ratio, lower resistance makes the phase plot larger. We observe that the inertial effects dominate at lower aspect ratios and their dominance reduces with increasing aspect ratio. This is also evident from the perturbation solution. We observe that the perturbation solutions show better agreement with the numerical solution at higher aspect ratios. 

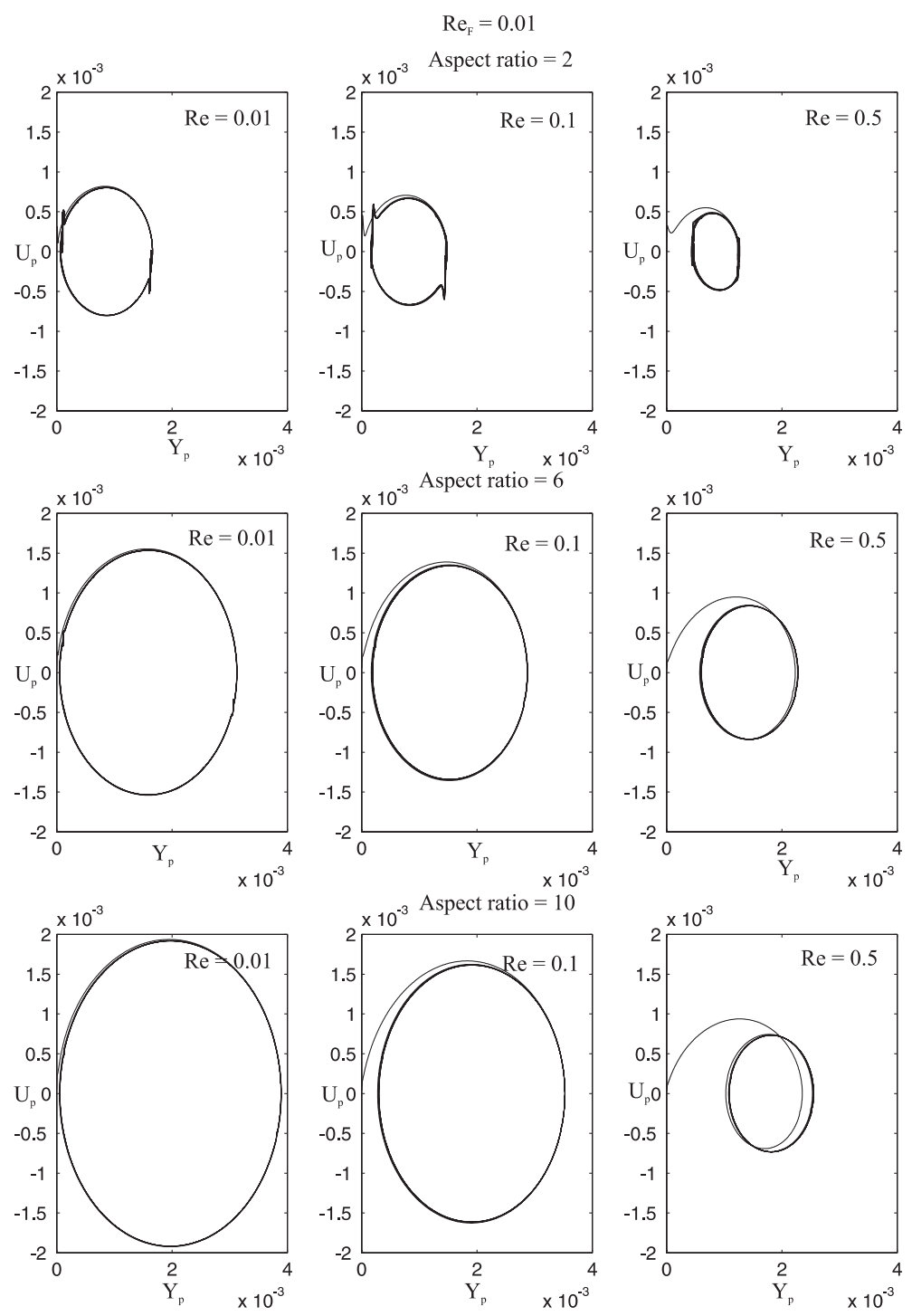

Figure 5. Phase portrait obtained for different $\operatorname{Re}$ values $(0 \cdot 01,0 \cdot 1,0.5), \operatorname{Re}_{F}=0.01$, aspect ratio $=2,6,10$. We can see the effect of increasing Re which results in diminished phase plots and also of the higher aspect ratio wherein the inertial effects are lower.

The attainment of an oscillatory steady state is found to be quicker when the aspect ratio is larger. This happens due to weaker inertial effects.

The values obtained for the acceleration term for different aspect ratios are given in table 1 . We see that the values of the second and third diagonal elements of the tensor are quite similar to one another. This is expected as both are symmetric to the direction of motion of particle, in the current work. These values give us an idea about the reaction to particle motion and hence the term acceleration reaction. As can be seen, the values increase with increasing aspect ratio. These values appear in the term Re' in the equation (6). This term additionally contains 
a factor which is the square of the inverse of aspect ratio. The contribution from the Pseudosteady Stokes drag also decreases with increasing aspect ratio which again contributes to lower resistance. The decrease of resistance with aspect ratio could be due to the body becoming more streamlined and hence being able to move freely through the fluid medium easily.

The effect of nonlinearity was evident when we took the power spectrum of the displacement time series. We observed that there exist subharmonics in the neighbourhood of $\operatorname{Re}_{F}=0.01$, which decreased with the increase in the aspect ratio and increased with the increase in the Re. There were some glimpses of higher harmonics which vanished with increase in aspect ratio.

In this regime we found the power spectrum of the displacement time series for different values of $\operatorname{Re}, \operatorname{Re}_{F}$ and aspect ratio. In figure 6 we have presented these results. Note that at
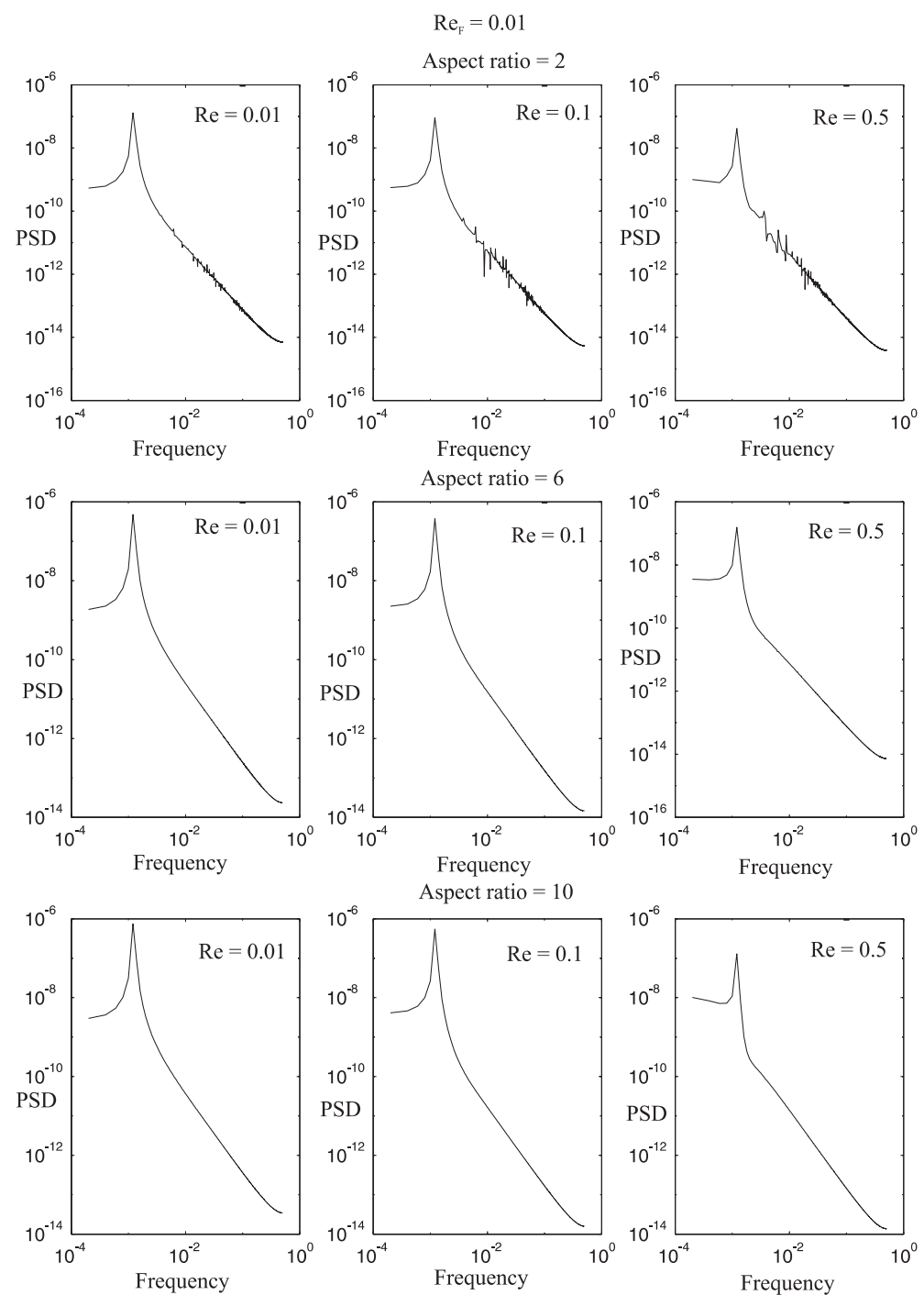

Figure 6. The power spectrum for $\operatorname{Re}_{F}=0 \cdot 01, \mathrm{Re}=0 \cdot 01,0 \cdot 1$ and 0.5 and aspect ratio 2,6 and 10 . We can see higher harmonics at low aspect ratio and $\operatorname{Re}_{F}=0 \cdot 01$, a clear effect of inertia. 


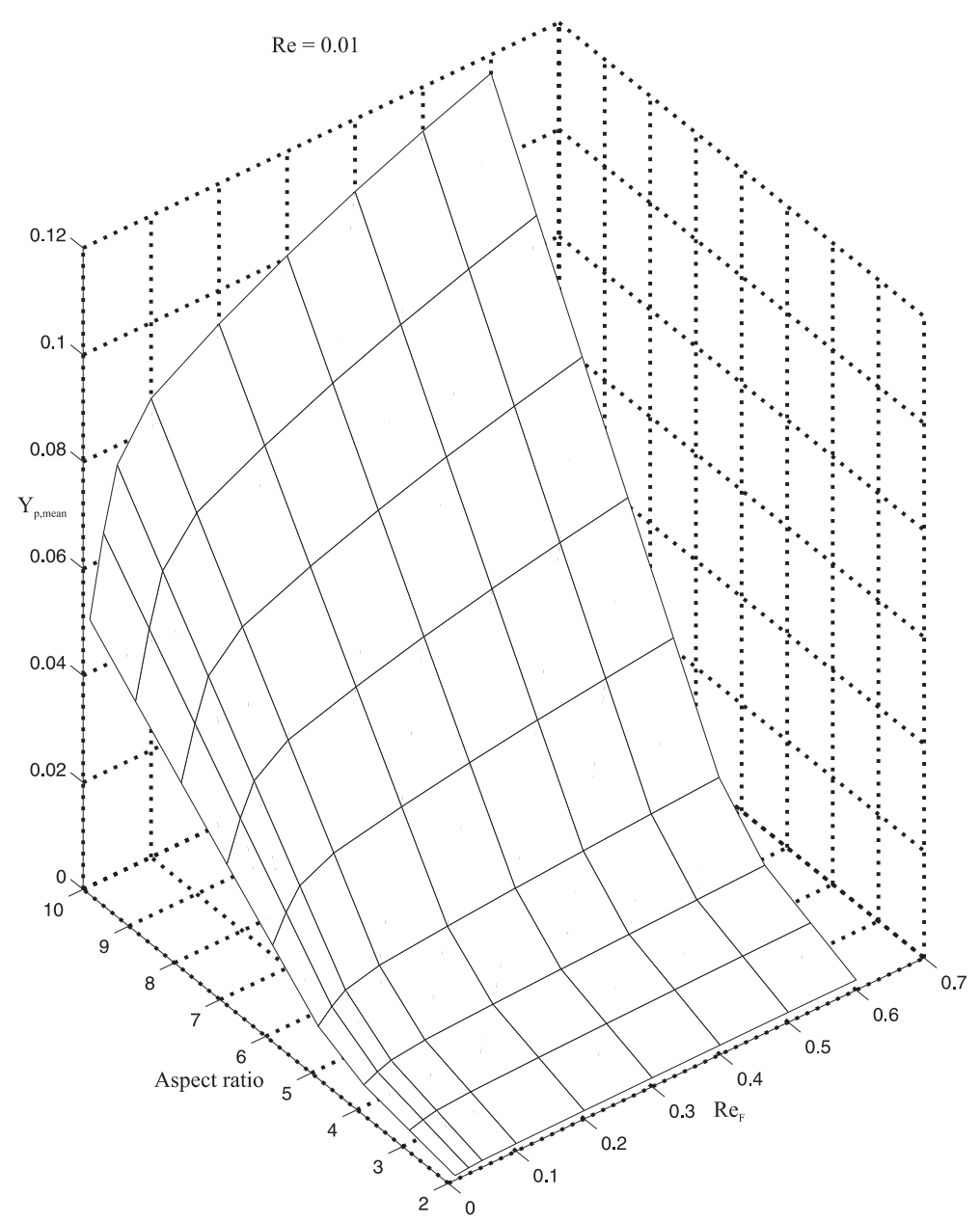

Figure 7. Typical plot showing the relationship of $Y_{\text {pmean }}$ with $\operatorname{Re}_{F}$ and aspect ratio for $\operatorname{Re}=0 \cdot 01$.

aspect ratio $=2$, there exist higher harmonics other than the fundamental dominant sinusoidal part. The amplitude of these higher harmonics increase with the increase in Re and hence inertia.

We observe that there exists a definite relation between $Y_{\text {pmean }}$ and $\mathrm{Re}$, as well as $Y_{\text {pmean }}$ and $\operatorname{Re}_{F}$. We see that as the quantities $\operatorname{Re}_{F}$ and $\operatorname{Re}$ increase, the value of $Y_{\text {pmean }}$ also increases. This variation is shown in figures 7 and 8 , respectively. These figures also show the effect of aspect ratio on $Y_{\text {pmean }}$ and find that $Y_{\text {pmean }}$ increases with the aspect ratio. We have presented a typical case in these figures. However, the pattern remains the same for all other cases.

When we apply a phase shift of $\pi$ to the sinusoidal forcing term the attractors shift their position about the velocity axis. That is, when we apply a force in an initially negative direction (the opposite direction) $Y_{p}$ shows a reflection about the $Y_{p}=0$ axis, namely we obtain a reflection of the attractor. Figure 9 shows the phase plots when the direction of the amplitude of the force is changed and the attractors form a reflection of each other about the axis $Y_{p}=0$, as expected. Since the direction of the force represents the direction of initial motion and also 


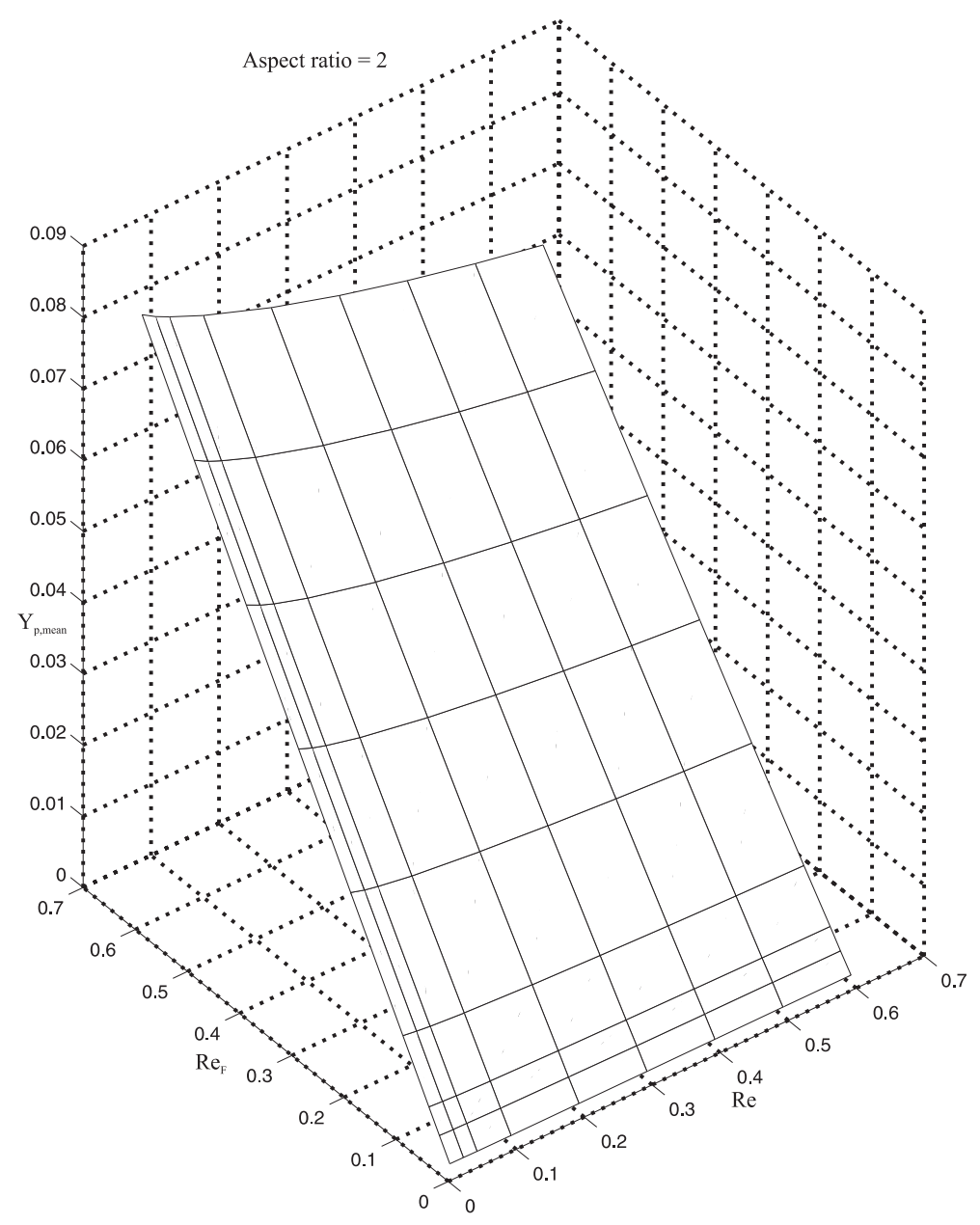

Figure 8. Typical plot showing the relationship of $Y_{\text {pmean }}$ with $\operatorname{Re}$ and $\operatorname{Re}_{F}$ for a particular aspect ratio $=2$.

there is a fading memory, the particle shows an initial displacement and at large times the periodic motion manifests itself.

The possibility of separating particles by shape has been investigated. In order to demonstrate this idea, a particle each of a given aspect ratio (2-10) is considered. The particle ensemble is assumed to be at rest, placed at $Y_{p}=0$ at time $t=0$. It is assumed that the major axes of the spheroids are lined up along a single direction. A periodic force is then applied to each individual particle along this direction. The displacement of every particle is tracked as the time progresses. We performed the simulation up to 5000 iterations. The simulation result after 5000 iterations has been presented in figure 10 for the parameter set, $\operatorname{Re}=0 \cdot 01$ and $\operatorname{Re}_{F}=0 \cdot 6$. We note that the position of particles has been strongly influenced by the aspect ratio of the particle. The particle with aspect ratio 10 shows the maximum displacement while the one with aspect ratio 2 shows the minimum displacement from the initial position (figure 10). This indicates that there exists a possibility of separating particles by shape. 

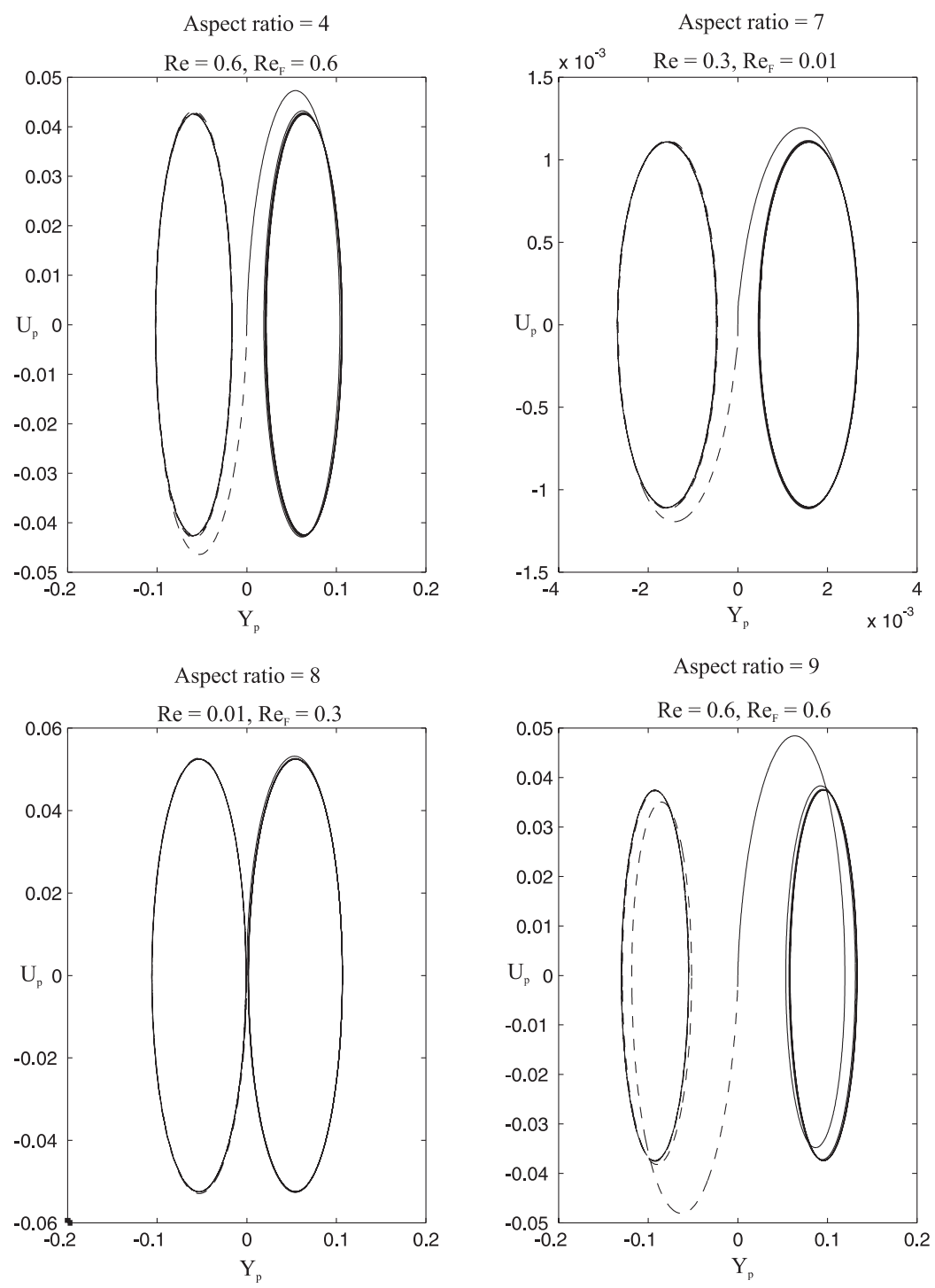

Figure 9. The phase portrait obtained at aspect ratio $=4, \operatorname{Re}=0 \cdot 6, \operatorname{Re}_{F}=+0.6$ and $-0 \cdot 6$, aspect ratio $=7, \operatorname{Re}=0 \cdot 3, \operatorname{Re}_{F}=+0 \cdot 01$ and -0.01 , aspect ratio $=8, \operatorname{Re}=0 \cdot 01, \operatorname{Re}_{F}=+0 \cdot 3$ and $-0 \cdot 3$ and aspect ratio $=9, \operatorname{Re}=0 \cdot 6, \operatorname{Re}_{F}=+0.6$ and -0.6 . This shows the reflection property of our solutions indicating that there exists a physical basis to our results.

We need to understand the effects of the individual particle dynamics in the system on a macroscopic experimentally accessible parameter, Here, we define a non-zero rheological parameter, namely a quantity analogous to the normal stress $\Sigma_{x x}^{p}$ for a dilute suspension of prolate spheroids. Batchelor (1970) developed a method for computing the bulk stress generated by a flowing suspension in terms of volume averages. In this formulation, we consider the suspension of particles in a quiescent Newtonian fluid. The volume fraction is taken to be small and hence no interaction between particles is assumed. Since the particle motion is 


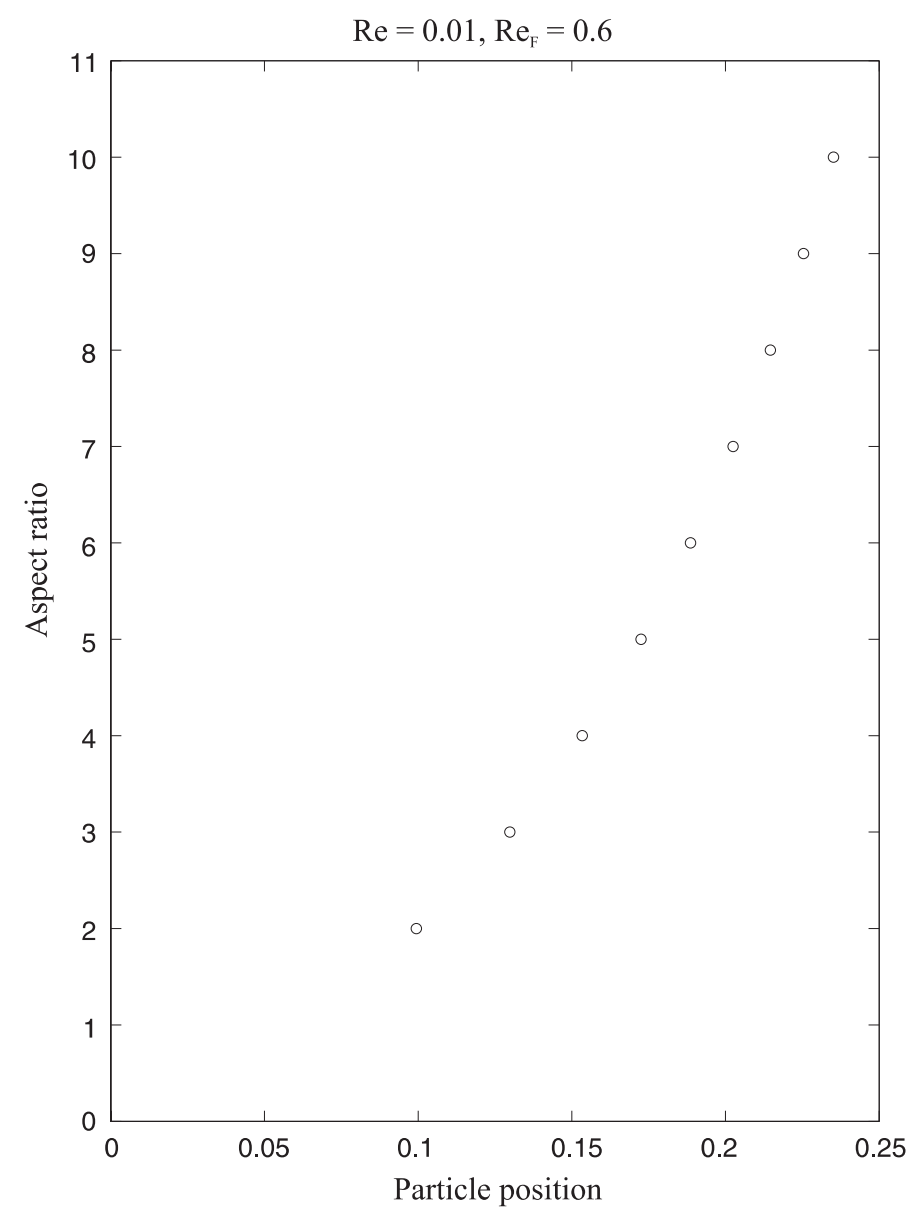

Figure 10. Plot showing the position of particles at the end of 5000 iterations for the parameter set, $\mathrm{Re}=0.01$ and $\operatorname{Re}_{F}=0 \cdot 6$. We see that the particle position is strongly influenced by the aspect ratio exhibiting a possibility of separating particles by shape.

approximately oscillatory, we assumed that there was no net motion of the particle through the fluid. Under these assumptions we get (Kulkarni \& Morris 2008)

$$
\Sigma^{P}=\frac{1}{V} \sum_{i} \boldsymbol{S}_{i}-\frac{\operatorname{Re}}{V} \sum_{i} \int_{V_{p}} \frac{1}{2}(\boldsymbol{a x}+\boldsymbol{x} \boldsymbol{a}) d V_{i}-\frac{\operatorname{Re}}{V} \int_{V} \boldsymbol{u}^{\prime} \boldsymbol{u}^{\prime} d V,
$$

where the first term represents the stresslet, or symmetric first moment of surface stress, exerted by a particle $i$, given for a rigid particle by

$$
\boldsymbol{S}_{i}=\int \frac{1}{2}(\boldsymbol{x} \boldsymbol{\sigma} \cdot \boldsymbol{n}+\boldsymbol{\sigma} \cdot \boldsymbol{n} \boldsymbol{x}) d A_{i} .
$$

Here $\boldsymbol{n}$ is the normal directed outward from the particle surface into the fluid phase. The second and the third terms in the expression of the particle stress denote the stress due to acceleration, given by $\boldsymbol{a}$ of the particles and the Reynolds stress, respectively. The expression 

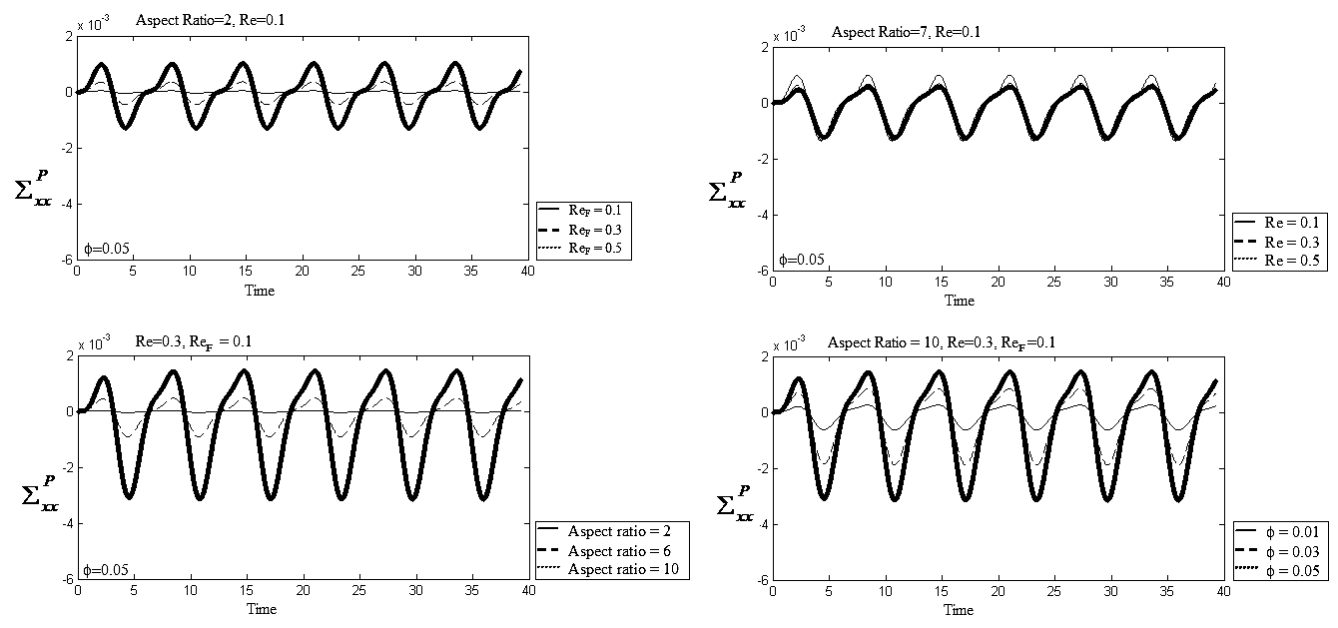

Figure 11. Plots showing the relationship between the 'normal stress' and $\operatorname{Re}_{F}(\mathbf{a}), \operatorname{Re}(\mathbf{b})$, aspect ratio (c) and volume fraction (d).

(8) directly shows that the contributions to the bulk stress from the acceleration and Reynolds stress are dependent on Re; the stresslet is dependent on Re through the flow field. For a dilute suspension, the interactions between the particles can be neglected and the solution for a single particle can be used to calculate the particle stress. For our problem, the particle stress $\Sigma_{x x}^{p}$ is found correct up to $\mathrm{O}(\mathrm{Re})$. We include only the zeroth order terms in the second and third terms on the R.H.S of equation (8) since our equations are correct only up to $\mathrm{O}(\mathrm{Re})$.

$$
\Sigma_{x x}^{p}=\frac{3}{4 \pi} \phi\left(\frac{a}{b}\right)^{2} Y_{p}(t) \operatorname{Re}_{F} \sin (t)-\frac{\operatorname{ReRe}_{F}^{2} \phi \cos (t)(1-\cos (t))}{36 \pi^{2}(c o f)^{2}}-\operatorname{Re} U_{p}^{2}
$$

For the Stokes' flow case the non-zero macroscopic parameter analogous to normal stress is given by

$$
\Sigma_{s t x x}=\frac{1}{8 \pi^{2}(\operatorname{cof})} \phi\left(\frac{a}{c}\right)^{2} \operatorname{Re}_{F}^{2} \sin (t)(1-\cos (t)) .
$$

We present here results obtained by varying the parameters involved in the expression. Figure 11 shows the variation of the 'normal stress' with different parameters. This parameter is easier to measure than the motion of the particle itself and hence, it can be used to correlate with system parameters. We varied the volume fraction $\phi, \operatorname{Re}, \operatorname{Re}_{F}$ and the aspect ratio and found that these parameters have a strong influence on the mean normal stress difference. We see that with increase in the volume fraction, the amplitude of the periodic force or the aspect ratio, the mean 'normal stress' shows an increasing trend, whereas with increasing Re, the value of the 'normal stress' decreases. Figure 12 shows the variation of 'normal stress' amplitude with volume fraction and aspect ratio. These results are likely to be quite important from a technological point of view and are also quite interesting as small changes in controllable parameters lead to relatively large changes in the 'normal stress'. We note that even in this simplified case, the suspension behaviour may be considered to be different from that of a typical suspension. Hence it is expected that the macroscopic parameters will be more complex when we study more complex situations. 


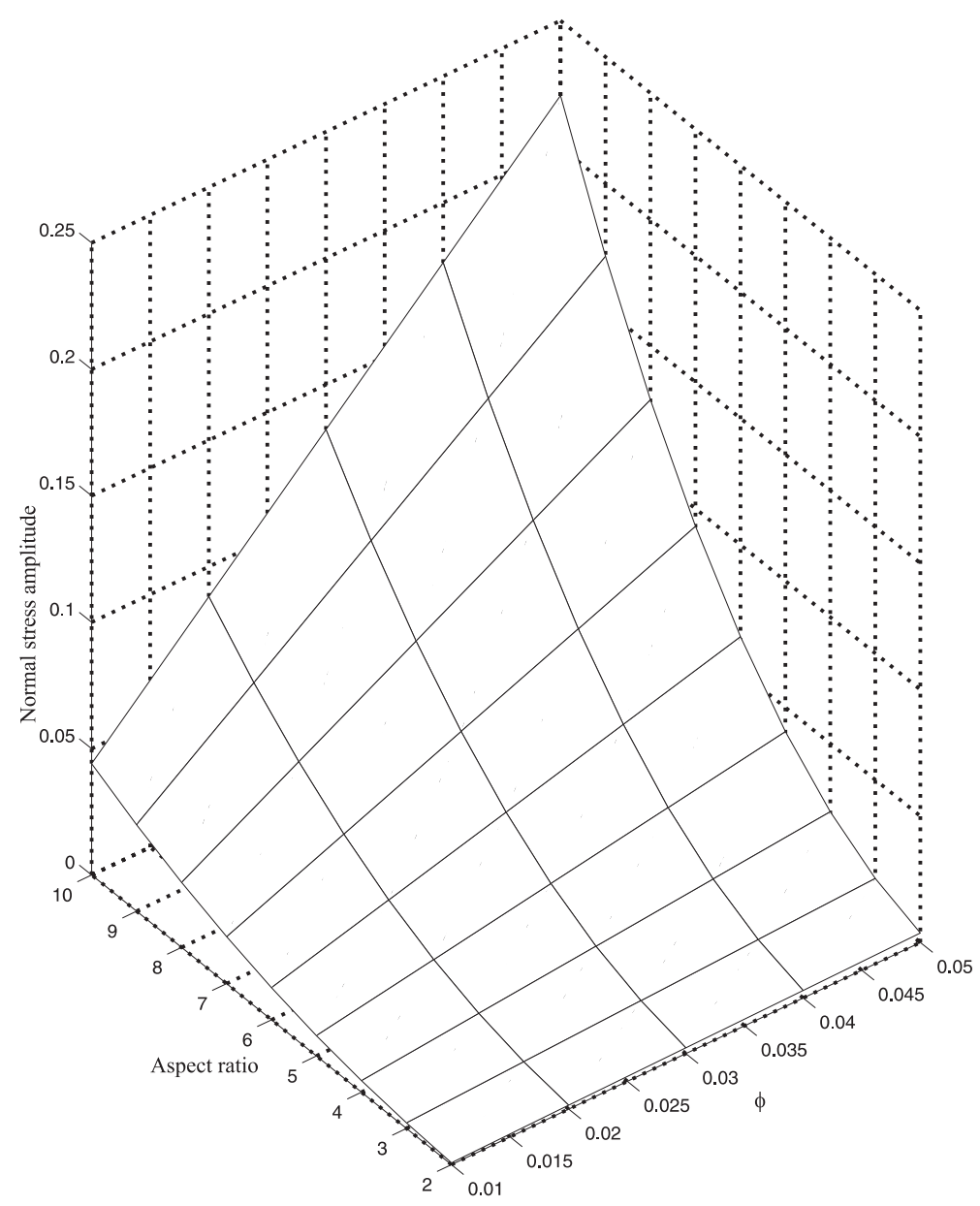

Figure 12. Plots showing relation between the 'normal stress' and aspect ratio and volume fraction.

\section{Conclusion}

In this paper, an attempt has been made to determine the dynamics and 'normal stress' of a dilute suspension of prolate spheroids under periodic forcing in a quiescent Newtonian fluid medium at low Reynolds numbers. The particle is seen to oscillate under periodic forcing. A preferred direction of motion is observed and it is seen that the particle shows a net displacement along this direction with time. The effect of system variables is studied in detail and it is found that increasing Re restricts the particle motion and hence the size of the attractor. Increasing the periodic force amplitude is found to increase the size of the attractor. The effect of the shape of the particle is studied by varying the aspect ratio and interesting results have been obtained. The size of the attractor increases with increasing aspect ratio. This result may be used to separate particles by shape. We have supplemented our results with detailed physical arguments and wherever possible, various tests have been conducted to justify our results. It is seen that the macroscopic parameter, the 'normal stress' is strongly dependent on system variables through their dependence on the expression in the 'normal 
stress' as well as the dependence on the individual particle motion. We find a number of interesting results in our analysis of the problem. This suggests that a large number of interesting features may be obtained in more complex flows and more complex suspensions. It is hoped that this work excites further research in this area.

The authors wish to acknowledge Dr. A R Upadhya, Scientist-in-Charge, CSIR-CMMACS, Bangalore 560037 and Prof. N. Rudraiah, Honorary Professor, UGC - CAS, Department of Mathematics, Bangalore University, Bangalore, for their kind encouragement. The authors also wish to acknowledge the Department of Science and Technology, Govt. of India, New Delhi for financial assistance vide Sanction Letter No. SR/S3/CE/33/2004-SERC Engg. One of the authors, K. Madhukar wishes to thank NAL/C-MMACS for providing the fellowship to do this research work. We also thank the three anonymous referees for their comments on our work, which helped us to improve the quality of the manuscript.

\section{List of symbols}

$\begin{array}{ll}\boldsymbol{F}^{H} & \text { hydrodynamic force (vector) } \\ \boldsymbol{F}_{\boldsymbol{s}}^{\boldsymbol{H}} & \text { Stokes expression for hydrodynamic force (vector) } \\ \boldsymbol{\phi} & \text { Stokes resistance tensor } \\ Y_{\mathrm{p}, \text { mean }} & \text { mean of the displacement of the particle } \\ \mathrm{Re} & \text { Reynolds' number } \\ \mathrm{S} 1 & \text { Strouhal number } \\ \Sigma_{x x}^{p} & \text { The xxth component of 'normal stress' difference } \\ \phi & \text { The volume fraction }\end{array}$

\section{References}

Asokan K, Kumar C V A, Dasan J, Radhakrishnan K, Kumar K S, Ramamohan T R 2005 Review of chaos in the dynamics and rheology of suspensions of orientable particles in simple shear flow subjected to an external periodic force. J. Non-Newtonian Fluid Mech. 129: 128-142

Batchelor G K 1970 The stress system in a suspension of force free particles. J. Fluid Mech. 41(3): 545-570

Bretherton F P 1962 The motion of rigid particles in a shear flow at low Reynolds numbers. J. Fluid Mech. 14: 284-304

Buevich Yu A, Syutkin S V, Tetyukhin V V 1984 Theory of a developed magnetofluidized bed. Magnetohydrodynamics (NY) 20: 333-339

Cebers A 1993 Chaos: New trend of magnetic fluid research. J. Magnetism and Magnetic Mater. 122: 281-285

Chwang A T, Wu T Y 1975 Hydromechanics of low-Reynolds-number flow, Part 2. Singularity method for Stokes flows. J. Fluid Mech. 67: 787-815

Gondret P, Lance M and Petit L 2002 Bouncing motion of spherical particles in fluids. Phys. Fluids 14(2): 643-652

Ignatenko N M, Melik-Gaikazyan Yu, Polunin V M, Tsebers A O 1984 Excitation of ultrasonic vibrations in a suspension of uniaxial ferromagnetic particles by volume magnetostriction. Magnetohydrodynamics (NY) 20: 237-240 
Jeffery G B 1922 The motion of ellipsoidal particles immersed in a viscous fluid. Proc. R. Soc. Lond. A102: 161-179

Kulkarni P M, Morris J F 2008 Suspension properties at finite Reynolds numbers from simulated shear flow. Phys. Fluids 20: 040602

Kumar C V A, Kumar K S, Ramamohan T R 1995 Chaotic dynamics of periodically forced spheroids in simple shear flow with potential application to particle separation. Rheol. Acta 34: 504-512

Kumar C V A, Ramamohan T R 1998 Controlling chaotic dynamics of periodically forced spheroids in simple shear flow: Results for an example of a potential application. Sädhanā 23: 131-149

Leal L G 1980 Particle motions in a viscous fluid. Ann. Rev. Fluid Mech. 12: 435-476

Lovalenti P M, Brady J F 1993 The hydrodynamic force on a rigid particle undergoing arbitrary timedependent motion at small Reynolds number. J. Fluid Mech. 256: 561-605

Lovalenti P M, Brady J F 1993a The force on a bubble, drop, or particle in arbitrary time-dependent motion at small Reynolds number. Phys. Fluids A5(9): 2104-2116

Lovalenti P M, Brady J F 1995 The temporal behaviour of the hydrodynamic force on a bofy in response to an abrupt change in velocity at small but finite Reynolds numbers. J. Fluid Mech. 293: $35-46$

Nayfeh A H 1973 Perturbation methods, A Wiley-Interscience publication

Press W H, Teukolsky S A, Vetterling W T, Flannery B P 1992 Numerical recipes in FORTRAN 77, second edition 1992. The art of scientific computing. Cambridge University Press

Pozrikidis C 1992 Boundary integral and singularity methods for linearized viscous flow, Cambridge University Press

Ramamohan T R, Shivakumara I S, Madhukar K 2009 Numerical simulation of the dynamics of a periodically forced spherical particle in a quiescent Newtonian fluid at low Reynolds numbers. Lect. Notes. Comp. Sci. 5544: 591-600

Rosensweig R E 1985 Ferrohydrodynamics, Cambridge University Press, Cambridge

Subramanian G, Koch D L 2006 Inertial effects on the orientation of nearly spherical particles in simple shear flow. J. Fluid Mech. 557: 257-296

Strand S R, Kim S 1992 Dynamics and rheology of a dilute suspension of dipolar nonspherical particles in an external field: Part 1. Steady shear flows. Rheologica Acta 34: 94-117

Tsebers A O 1986 Numerical modelling of the dynamics of a drop of magnetizable liquid in constant and rotating magnetic fields. Magnetohydrodynamics 22: 345-351 